\title{
Friction drag reduction achievable by near-wall turbulence manipulation in spatially developing boundary-layer
}

\author{
Amaury Bannier, ${ }^{1}$ Eric Garnier, ${ }^{1, a)}$ and Pierre Sagaut ${ }^{2}$ \\ ${ }^{1}$ Applied Aerodynamics Department, ONERA-The French Aerospace Lab, \\ 92190 Meudon, France \\ ${ }^{2}$ Aix-Marseille Université, CNRS, Centrale Marseille, M2P2 UMR 7340, \\ 13451 Marseille, France
}

\begin{abstract}
Various control strategies, such as active feedback control or riblets, end up restraining near-wall turbulence. An analytical study is conducted to estimate the drag-reduction achievable by such control in zero-pressure-gradient turbulent boundary-layers. Based on an idealized control which damps all fluctuations $w$ ithin a near-wall layer, a composite flow $\mathrm{p}$ rofile is es tablished. It le ads to ex plicit mo dels for both the drag-reduction and the boundary-layer development rate. A skin-friction decomposition is applied and gives physical insights on the underlying phenomena. The control is found to alter the spatial development of the boundary-layer, resulting in detrimental impact on the skin-friction. However, the drag-reducing mechanism, attributed to the turbulence weakening, is found predominant and massive drag reductions remain achievable at high Reynolds number, although a minute part of the boundary-layer is manipulated. The model is finally assessed against Large Eddy Simulations of riblet-controlled flow.
\end{abstract}

\section{INTRODUCTION}

Engineering devices aiming at manipulating turbulence have been developed and studied for long. The benefits being sought range from drag reduction to heat transfer regulation and reactant mixing speedup. Environmental regulation policies, especially on the aeronautical industry, will maintain the interest on this field for decades.

Among others, riblets, ${ }^{1,2}$ hydrophobic surfaces, ${ }^{3,4}$ and active feedback control ${ }^{5,6}$ are diverse strategies intensively studied so far. Behind those very distinct methods, a common physical feature: they all end up affecting near-wall turbulence. Some promising active feedback control strategies are tuned to suppress, or at least ease, turbulent fluctuations within a given near-wall layer or at a particular elevation. ${ }^{7}$ Above riblets, the flow is well approached by the canonical turbulent one placed over a laminar near-wall layer in which no turbulent fluctuations are assumed to exist. ${ }^{8}$ Super-hydrophobic walls allowing a mean slip $u_{\text {slip }}$ in the streamwise direction lead to canonical mean and turbulent profiles ${ }^{3}$ when the mean velocity is scaled as $u^{+}-u_{\text {slip }}^{+}$. This boosts the mean velocity, and, therefore, reduces turbulence intensities at a given flow rate. Hence, it appears that very-localized actions at the wall or in the near-wall region can successfully achieve substantial skin-friction reduction.

Fukagata, Iwamoto, and Kasagi ${ }^{9}$ (FIK hereafter) split the skin-friction into several contributing terms according to their physical underlying origins. Their decomposition allows a spatial localization of the contributing terms' intensity. Results evidence that, as the Reynolds number of the flow increases, a growing part of the friction can be attributed to the turbulence through the whole boundary-layer extent. ${ }^{10}$

a) eric.garnier@onera.fr 
Those two last conclusions seem contradictory: how can a near-wall localized control achieve substantial drag reduction while a major part of the friction tends to be attributed to turbulent mechanisms occurring further from the wall?

Iwamoto et al. ${ }^{11}$ led an analytical development to clarify the dilemma and quantified the drag reduction achievable by near-wall manipulation at high Reynolds number. Their analysis has been conducted in the frame of turbulent channel flow, in which no spatial development occurs. However, in most of external flow applications, the boundary-layer thickening has a major influence on the drag. Indeed, the thicker the boundary-layer, the lower the drag, while the lower the drag, the lower the boundary-layer thickening rate. Drag-reducing control devices indirectly slow down the boundary-layer development, which has a detrimental effect on the drag. This secondary effect, not present in channel flow, is inherent to spatially developing turbulent boundary-layers. Whether or not it can become predominant over the beneficial drag-reduction could have important practical consequences.

The present study aims at investigating the influence of near-wall turbulence manipulation on both the spatial development and the skin-friction of zero-pressure-gradient turbulent boundarylayers (ZPGTBLs). Following Iwamoto et al. ${ }^{11}$ near-wall turbulent fluctuations are supposed to be totally damped by a control strategy considered as ideal. Direct drag-reducing effects, comparable to those observed in the channel flow case, are expected to be retrieved, but must appear along with additional repercussions due to the spatially developing nature of ZPGTBLs.

Assumptions behind the use of an "ideal" control constitute the basis of the present analytical development and are detailed in Sec. II. The direct drag-reducing effect of the control is retrieved in Sec. III while its influence on the boundary-layer development comes into Sec. IV. Later, in Sec. V, both effects are simultaneously taken into account and reveal the performance actually achievable by such a near-wall turbulence manipulation. More insight is given in Sec. VI by considering the impact of the control on each of the FIK decomposition terms. At last, the proposed model is assessed versus Large Eddy Simulation (LES) results.

\section{A COMPOSITE MODEL FOR THE CONTROLLED FLOW}

The ideal control considered in the present study is assumed to damp all turbulent fluctuations within a thin near-wall layer of thickness $\Delta y^{+}$. The superscript + denotes quantities normalized in inner (or viscous) scaling, that is, using the viscosity $v$ and the local friction velocity $u_{\tau}=\sqrt{\tau_{w} / \rho}$, where $\tau_{w}$ and $\rho$ are the wall shear-stress and the fluid density. Within the damped layer, the total shear-stress reduces to its viscous contribution, which is assumed constant, leading to

$$
\begin{aligned}
& u^{+}\left(y^{+}<\Delta y^{+}\right)=y^{+}, \\
& R_{i j}^{+}\left(y^{+}<\Delta y^{+}\right)=0,
\end{aligned}
$$

where $u$ stands for the mean velocity and $R_{i j}$ for the Reynolds stress tensor (in particular, the Reynolds shear-stress is denoted $R_{12}$ or $R_{x y}$ ). At the upper boundary $y^{+}=\Delta y^{+}$of the controlled layer, the inner-scaled velocity equals $u^{+}=\Delta u^{+}=\Delta y^{+}$and the shear-stress is conserved from the wall: $\tau=\tau_{w}$.

Above the controlled layer, turbulent fluctuations are not damped anymore. When considered in the local frame of reference moving at $u^{+}=\Delta y^{+}$and with a wall-normal origin located at $y^{+}=\Delta y^{+}$, the superimposed turbulent flow is denoted with a prime,

$$
\begin{aligned}
y^{+} & =\Delta y^{+}+y^{\prime+}, \\
u^{+}\left(y^{+}>\Delta y^{+}\right) & =\Delta y^{+}+u^{\prime+}\left(y^{\prime+}\right), \\
R_{i j}^{+}\left(y^{+}>\Delta y^{+}\right) & =R_{i j}^{\prime+}\left(y^{\prime+}\right) .
\end{aligned}
$$

Taken together, the "inner-layer viscous profile" below $y^{+}=\Delta y^{+}$, Eqs. (1), and the "superimposed turbulent flow" above it, Eqs. (2), define the "composite profile" that characterizes the controlled flow. It is represented in Fig. 1 . The inner scaling is unambiguously defined since $u_{\tau}^{\prime}=u_{\tau}$, resulting from $\tau_{w}^{\prime}=\tau\left(y^{+}=\Delta y^{+}\right)=\tau_{w}$. However, outer scaling and Reynolds number definitions must be 


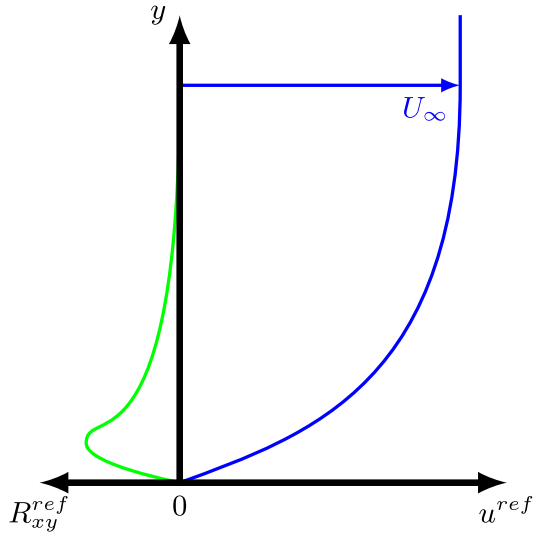

(a)

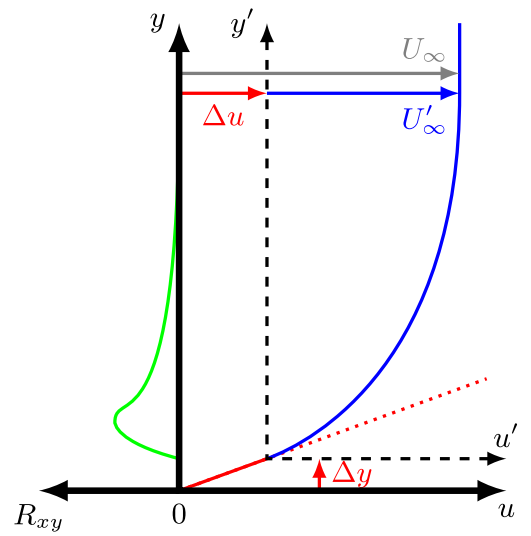

(b)

FIG. 1. Schematic mean velocity and Reynolds shear stress profiles. (a) Uncontrolled canonical ZPGTBL profiles. (b) Controlled composite profiles.

treated with care because the free-stream velocities of the composite and superimposed flows differ: $U_{\infty}^{+}=\Delta y^{+}+U_{\infty}^{\prime+}$.

The mean and turbulent profiles $u^{\prime+}\left(y^{\prime+}\right)$ and $R_{i j}^{\prime+}\left(y^{\prime+}\right)$ of the superimposed turbulent flow are assumed to be those of an uncontrolled canonical ZPGTBL flow, denoted by the superscript ref,

$$
\begin{aligned}
& u^{\prime+}\left(y^{\prime+}\right)=u^{r e f+}\left(y^{+}\right), \\
& R_{i j}^{\prime+}\left(y^{\prime+}\right)=R_{i j}^{r e f+}\left(y^{+}\right) .
\end{aligned}
$$

This assumption is quite strong, and one can wonder whether the above-defined composite profile with a given $\Delta y^{+}$relevantly characterizes the flow that would be effectively obtained by damping near-wall fluctuations or by applying any specific control device (opposition control, riblets, super-hydrophobic wall, etc.). In this last case, the question of the definition of an equivalent damped layer thickness $\Delta y^{+}$also arises.

In practice, depending on the nature of the control device, the equivalent thickness $\Delta y^{+}$is determined from flow conditions and control parameters. For instance, active feedback control aims at damping fluctuations within a near-wall layer of specified thickness, thus, the definition of $\Delta y^{+}$is straight-forward. For small enough riblets-that is riblets in their drag-reducing viscous-regime ${ }^{2}-\Delta y^{+}$is found proportional to the riblet size and can be estimated empirically from the riblet design. ${ }^{2}$ Unfortunately, the proportionality breaks when riblets become taller, ${ }^{12}$ so $\Delta y^{+}$cannot be increased infinitely. Flows bounded by hydrophobic walls are not accurately described by the composite profile and a model with $y^{+}=y^{\prime+}$ instead of (2a) would be better suited. However, for most of the following conclusions, $\Delta y^{+}$can be considered negligible against $\delta^{+}$, so no significant quantitative difference has to be noted. In this case, $\Delta y^{+}$corresponds to the mean streamwise slip length. ${ }^{3}$ More generally, as long as a control device satisfies the composite profile, its value for $\Delta y^{+}$can be computed a posteriori from the observed drag-reduction $D R_{\tau}=\left(c_{f}^{r e f}\left(R e_{\tau}\right)-c_{f}\left(R e_{\tau}\right)\right) / c_{f}^{r e f}\left(R e_{\tau}\right) \mathrm{using}^{8}$

$$
\Delta y^{+}=\sqrt{\frac{2}{c_{f}^{r e f}}}\left(\frac{1}{\sqrt{1-D R_{\tau}}}-1\right) .
$$

Fig. 2 shows the mean velocity and turbulent intensity profiles of flat-plate and riblet bounded flows obtained by LES. ${ }^{8}$ Above the 8-wall-unit-tall trapezoidal riblets, the equivalent $\Delta y^{+}$is found equal to 1.4. Both actual and "superimposed" profiles, obtained using (2), have been plotted aside flat-plate reference ones. Superimposed profiles $u^{\prime+}\left(y^{\prime+}\right)$ and $R_{i j}^{\prime+}\left(y^{\prime+}\right)$ perfectly collapse with $u^{r e f+}\left(y^{+}\right)$and $R_{i j}^{r e f+}\left(y^{+}\right)$, at same Reynolds number $\delta^{++}=\delta^{r e f+}$. Later, in Sec. VII, additional comparisons with the riblet-controlled flow will be performed for an a posteriori validation of the analytic development. A similar result can be evidenced using the mean and turbulent profiles from 


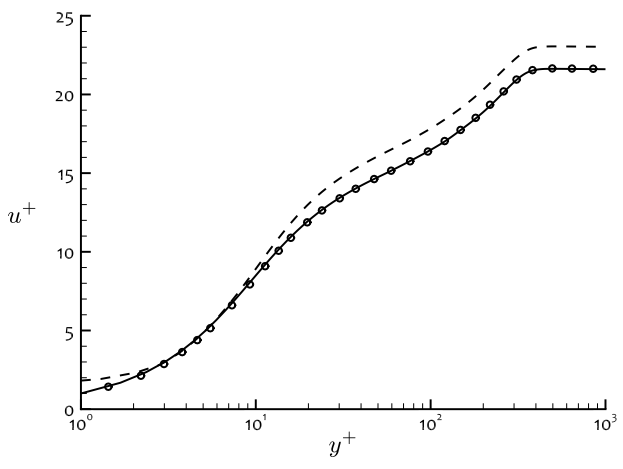

(a)

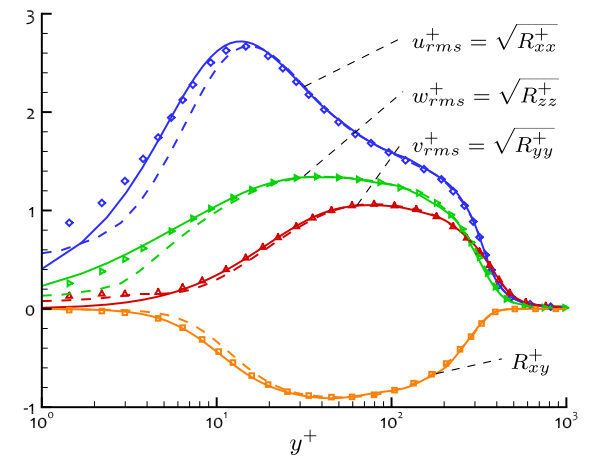

(b)

FIG. 2. (a) Mean velocity and (b) turbulence intensity profiles scaled in wall-units: (solid line) reference canonical profiles $u^{r e f+}\left(y^{+}\right)$and $R_{i j}^{r e f+}\left(y^{+}\right)$at Reynolds number $\delta^{r e f+}=360$; (dashed line) actual riblet profiles $u^{+}\left(y^{+}\right)$and $R_{i j}^{+}\left(y^{+}\right)$; (symbols) superimposed profiles $u^{\prime+}\left(y^{\prime+}\right)$ and $R_{i j}^{\prime+}\left(y^{\prime+}\right)$ at Reynolds number $\delta^{\prime+}=360$ obtained after shifting the actual riblet profiles by $\Delta y^{+}=1.4$ according to Eq. (2).

opposition control direct numerical simulation (DNS). ${ }^{7}$ This demonstrates the relevancy of the composite profile to characterize realistic controlled ZPGTBLs. Based on pioneering DNSs of active feedback control,,${ }^{5,13,14}$ Iwamoto et al. ${ }^{11}$ reached a similar conclusion for controlled channel flows.

\section{DIRECT EFFECT: SKIN-FRICTION REDUCTION}

Under the effect of the previously defined ideal control, which suppresses all turbulent fluctuations within a thin layer of thickness $\Delta y^{+}$, the altered flow field has been assumed well characterized by composite profiles. How does it influence the drag?

The skin-friction coefficient $c_{f}$ of the composite flow is given by

$$
\frac{c_{f}}{2}=\frac{\tau_{w}}{\rho U_{\infty}^{2}}=\frac{1}{U_{\infty}^{+2}}=\frac{1}{\left(U_{\infty}^{\prime+}+\Delta y^{+}\right)^{2}} .
$$

To relate $c_{f}$ to the flow Reynolds number, $U_{\infty}^{\prime+}$ must be made more explicit. Assumption (3) allows the use of canonical ZPGTBL relations for the superimposed turbulent flow, and especially, the Coles-Fernholz relation ${ }^{15}$

$$
\sqrt{\frac{2}{c_{f}^{r e f}}}=U_{\infty}^{r e f+}=\frac{1}{\kappa} \ln \left(R e_{\theta}^{r e f}\right)+C,
$$

where $R e_{\theta}=\theta U_{\infty} / v$ is the Reynolds number based on the momentum thickness $\theta=\int_{y=0}^{\infty} \frac{u(y)}{U_{\infty}}$ $\left(1-\frac{u(y)}{U_{\infty}}\right) \mathrm{d} y$. Coefficients $\kappa=0.384$ and $C=4.127$ have been recently corrected. ${ }^{16}$ This relation applies to high enough Reynolds number flows, imposing restrictions on the superimposed turbulent flow. Consequently, only results for which $R e_{\theta^{\prime}}^{\prime}=\theta^{\prime} U_{\infty}^{\prime} / v>450$ are considered in the present study. This threshold is low, since a flow at such a Reynolds number does not have the properties of canonical high Reynolds number flows, though the present theory will be shown to still yield valid results even at this low Reynolds number limit.

From definitions (1) and (2) of the composite mean velocity profile, $R e_{\delta_{1}}=\delta_{1} U_{\infty} / v$ and $R e_{\delta_{1}^{\prime}}^{\prime}=$ $\delta_{1}^{\prime} U_{\infty}^{\prime} / v$ on the one hand, and $R e_{\theta}=\theta U_{\infty} / v$ and $R e_{\theta^{\prime}}^{\prime}=\theta^{\prime} U_{\infty}^{\prime} / v$ on the other hand, can be related by

$$
\begin{array}{r}
\operatorname{Re}_{\delta_{1}}= \\
\operatorname{Re}_{\delta_{1}^{\prime}}^{\prime}+\left(1-\frac{1}{2} \frac{\Delta y^{+}}{U_{\infty}^{+}}\right) U_{\infty}^{+} \Delta y^{+}, \\
\operatorname{Re}_{\theta}=\left(1+\left(H^{\prime}-1\right) \frac{\Delta y^{+}}{U_{\infty}^{+}}\right) \operatorname{Re}_{\theta^{\prime}}^{\prime}+\left(1-\frac{2}{3} \frac{\Delta y^{+}}{U_{\infty}^{+}}\right) \frac{\Delta y^{+2}}{2},
\end{array}
$$


where $H^{\prime}=\delta_{1}^{\prime} / \theta^{\prime}$ is the shape factor of the superimposed turbulent flow. It can be estimated using the estimation provided by Nagib et al. ${ }^{16}$ for canonical ZPGTBL,

$$
H^{\text {ref }}\left(U_{\infty}^{\text {ref }+}\right)=\frac{1}{1-7.135 / U_{\infty}^{\text {ref }+}}=1+O\left(\frac{7.135}{U_{\infty}^{\text {ref }+}}\right) .
$$

Substitution of (5) and (6) into (8) yields an explicit formula between the Reynolds number $\operatorname{Re}_{\theta}$ and the friction coefficient (through $U_{\infty}^{+}=\sqrt{2 / c_{f}}$ ) of the composite flow,

$$
\operatorname{Re}_{\theta}=\left(1+\left(H^{r e f}\left(U_{\infty}^{+}-\Delta y^{+}\right)-1\right) \frac{\Delta y^{+}}{U_{\infty}^{+}}\right) e^{\kappa\left(U_{\infty}^{+}-\Delta y^{+}-C\right)}+\left(1-\frac{2}{3} \frac{\Delta y^{+}}{U_{\infty}^{+}}\right) \frac{\Delta y^{+2}}{2} .
$$

If $\Delta y^{+}$is set to 0 , the identity gets back to original Coles-Fernholz correlation (6). Else, if $\Delta y^{+}$ is assumed negligible against both $U_{\infty}^{+2}$ and $\sqrt{R e_{\theta}}$ as a first order approximation, the following modified Coles-Fernholz relation is obtained:

$$
\sqrt{\frac{2}{c_{f}}}=U_{\infty}^{+} \approx \frac{1}{\kappa} \ln \left(R e_{\theta}\right)+C+\Delta y^{+} .
$$

This approximate relation is rather precise as shown in Fig. 3(a), in which the influence of $\Delta y^{+}$on the skin-friction coefficient $c_{f}$ is plotted against $\operatorname{Re}_{\theta}$.

A drag reduction rate $D R_{\theta}$ can be computed by comparing the friction coefficients $c_{f}$ of the controlled and reference cases at the same Reynolds number $R e_{\theta}$,

$$
\begin{aligned}
D R_{\theta}\left(R e_{\theta}\right) & =\frac{c_{f}^{r e f}\left(R e_{\theta}\right)-c_{f}\left(R e_{\theta}\right)}{c_{f}^{r e f}\left(R e_{\theta}\right)}=1-\left(\frac{U_{\infty}^{r e f+}\left(R e_{\theta}\right)}{U_{\infty}^{+}\left(R e_{\theta}\right)}\right)^{2}, \\
& \approx 1-\left(1+\frac{\Delta y^{+}}{\frac{1}{\kappa} \ln \left(\operatorname{Re}_{\theta}\right)+C}\right)^{-2},
\end{aligned}
$$

where both original and modified Coles-Fernholz friction laws (6) and (11) have been used to obtained Eq. (13).

Fig. 3(b) shows the influence of $\Delta y^{+}$on $D R_{\theta}\left(R e_{\theta}\right)$. The drag reduction achieved by damping fluctuations in the - although extremely thin - near-wall part of the boundary-layer can be massive. For instance, at $\operatorname{Re}_{\theta}=10^{5}$, the boundary-layer thickness of the uncontrolled flow is about $\delta_{99}^{r e f+} \approx 3 \cdot 10^{4}$, meaning that $\Delta y^{+}=10$ is less than a thousandth of the boundary-layer. Yet, the damping of the fluctuations in this minute layer results in a substantial $40 \%$ drag reduction.

The control efficiency for a given $\Delta y^{+}$decreases when the Reynolds number $R e_{\theta}$ increases. However, the dependency is weak and significant drag reductions are still achievable even at large Reynolds number.

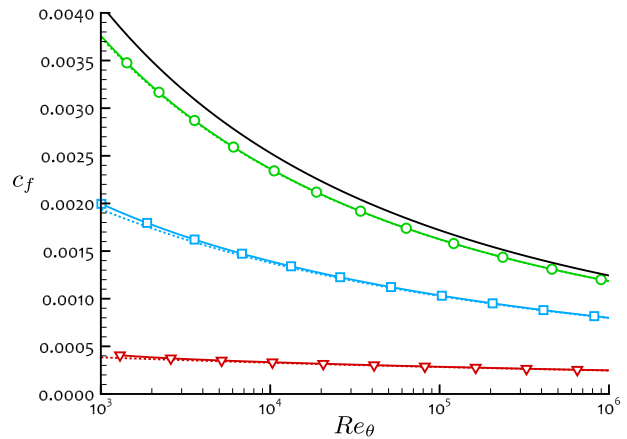

(a)

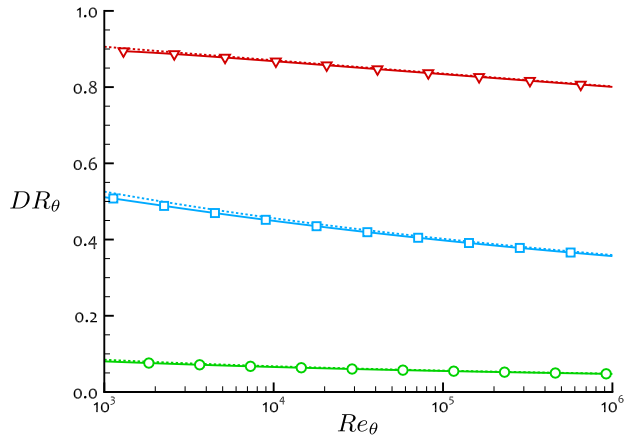

(b)

FIG. 3. Influence of the damped layer thickness $\Delta y^{+}$on (a) the skin-friction coefficient $c_{f}$ and (b) the drag reduction rate $D R_{\theta}$ versus $R e_{\theta}$ : (black solid line) no control; (green solid line, circle) $\Delta y^{+}=1$; (blue solid line, square) $\Delta y^{+}=10$; (red solid line, triangle) $\Delta y^{+}=30$. First-order approximations obtained from (11) and (13) are plotted with dotted lines aside the corresponding exact solutions. 
The drag reduction $D R_{\theta}\left(R e_{\theta}\right)$ observed here in a controlled ZPGTBL is closely similar to what is obtained in a channel flow on which the same near-wall fluctuation damping is applied. With $\Delta y^{+}=10$, Iwamoto et al. ${ }^{11}$ reports a $37 \%$ drag reduction for turbulent channel flow at $R e_{\tau}^{\text {ref }}=$ $\delta^{r e f+}=3 \cdot 10^{4}$. This value is consistent with the $40 \%$ obtained here in a spatially developing boundary-layer.

\section{INDIRECT EFFECT: DECREASE OF THE BOUNDARY-LAYER THICKENING RATE}

Unlike channel or pipe flows, spatially developing boundary-layers are not statistically homogeneous in the streamwise direction: they develop, thicken, and their Reynolds numbers increase. Their development rate is related to the skin-friction through the momentum-integral equation. ${ }^{17}$ In the present case of two-dimensional zero-pressure-gradient boundary-layer, this relation is simply formulated by

$$
\frac{\mathrm{d} \theta}{\mathrm{d} x}=\frac{c_{f}}{2}=\frac{1}{U_{\infty}^{+2}}
$$

Because $c_{f}$ is greatly reduced by the control, the streamwise thickening rate of the boundary-layer is expected to be decreased. Eq. (14) can be integrated to relate the physical streamwise location $x$ to the momentum thickness $\theta$

$$
R e_{x}\left(R e_{\theta}\right)=R e_{x}^{o f f s e t}+\int^{R e_{\theta}} U_{\infty}^{+2} \mathrm{~d} R e_{\theta} .
$$

For uncontrolled flows, after substitution of skin-friction Coles-Fernholz relation (6) into (15), the streamwise development of the boundary-layer thickness can be solved analytically ${ }^{16}$ and yields

$$
\operatorname{Re}_{x}^{r e f}\left(\operatorname{Re}_{\theta}\right)=\frac{1}{\kappa^{2}}\left(\left(\ln \left(\operatorname{Re}_{\theta}\right)+\kappa C-1\right)^{2}+1\right) R e_{\theta},
$$

where the integration offset $R e_{x}^{o f f s e t, r e f}$ has been arbitrarily set to 0 which indirectly set the $x$-origin.

For controlled flows, the relation $U_{\infty}^{+}\left(R e_{\theta}\right)$ is implicitly given by relation (10). Because no explicit form can be derived, an analytic expression is inaccessible. Numerical integration must be performed to express the evolution of $R e_{x}$ with respect to $R e_{\theta}$. However, if one used approximated law (11) instead of (10), analytical solving yields

$$
\operatorname{Re}_{x}\left(\operatorname{Re}_{\theta}\right) \approx \operatorname{Re}_{x}^{o f f s e t}+\frac{1}{\kappa^{2}}\left(\left(\ln \left(\operatorname{Re}_{\theta}\right)+\kappa C+\kappa \Delta y^{+}-1\right)^{2}+1\right) R e_{\theta},
$$

where $R e_{x}^{o f f s e t}$ is to be adjusted such that $\operatorname{Re}_{x}\left(R e_{\theta}^{i n i}\right)=\operatorname{Re}_{x}^{r e f}\left(R e_{\theta}^{i n i}\right)$ with $R e_{\theta}^{i n i}$ the Reynolds number at the location where the control starts. That is,

$$
\operatorname{Re}_{x}^{\text {offset }}\left(\operatorname{Re}_{\theta}^{i n i}\right)=-2\left(\frac{1}{\kappa} \ln \left(\operatorname{Re}_{\theta}^{i n i}\right)+C+\frac{\Delta y^{+}}{2}-\frac{1}{\kappa}\right) \Delta y^{+} \operatorname{Re}_{\theta}^{i n i} .
$$

Fig. 4(a) shows the streamwise development of the boundary-layer thickness $R e_{\theta}$ for different damped layer thicknesses $\Delta y^{+} \in\{1,10,30\}$ and for control applied from different locations $R e_{x}^{i n i} \in$ $\left\{10^{6}, 10^{7}, 10^{8}\right\}$ (corresponding to $\operatorname{Re}_{\theta}^{i n i} \in\left\{2.1 \cdot 10^{3}, 1.4 \cdot 10^{4}, 1.0 \cdot 10^{5}\right\}$. As expected, the growth rate of the boundary-layer is decreased by the control. For instance, with $\Delta y^{+}=10$ and $R e_{x}^{i n i}=10^{6}$, the controlled boundary-layer thickness at physical location $R e_{x}=10^{7}$ is reduced by more than a third $\left(R e_{\theta}=9.0 \cdot 10^{3}\right)$ compared to the reference case $\left(R e_{\theta}^{r e f}=1.4 \cdot 10^{4}\right)$. While the dependence of $\Delta y^{+}$on the boundary-layer thickening remains high, the influence of the control starting location $R e_{x}^{i n i}$ tends to soften downstream a transient of about a decade. In other words, from the location $R e_{x} \approx 10 R e_{x}^{i n i}$, the momentum thickness becomes comparable to the one that would have been obtained with a control applied from any earlier location and, in particular, from the very beginning of the plate. The boundary-layer thickening of this limiting case, for which $R e_{x}^{i n i}$ and $R e_{\theta}^{i n i}$ approach zero, is obtained by setting $R e_{x}^{o f f s e t}=0$ in relation (17). After the decade-long declining transient, the momentum thickness ratio $R e_{\theta} / R e_{\theta}^{r e f}$ starts increasing very slightly, so that it can be considered as roughly constant over the wide range of Reynolds number displayed in Fig. 4(b). In 


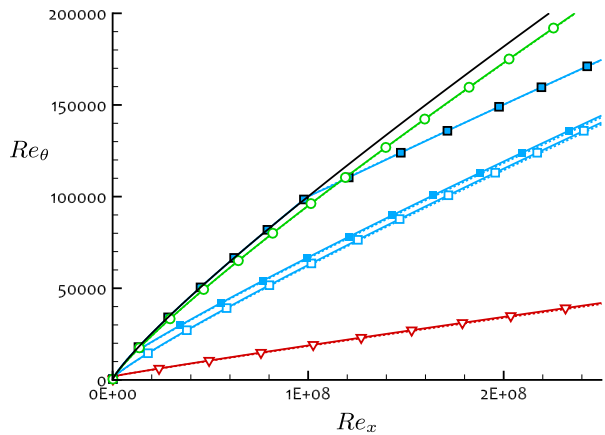

(a)

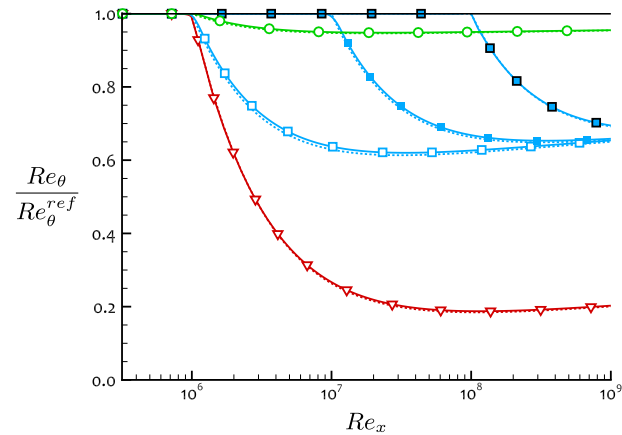

(b)

FIG. 4. Influence of both the damped layer thickness $\Delta y^{+}$and the control starting location $R e_{x}^{i n i}$ on (a) the momentum thickness Reynolds number $R e_{\theta}$ and (b) its ratio to the reference uncontrolled one $R e_{\theta} / R e_{\theta}^{r e f}$ : (black solid line) no control; (green solid line, empty circle) $R e_{x}^{i n i}=10^{6}, \Delta y^{+}=1$; (blue solid line, empty square) $R e_{x}^{i n i}=10^{6}, \Delta y^{+}=10$; (blue solid line, filled square) $R e_{x}^{i n i}=10^{7}, \Delta y^{+}=10$; (blue solid line, box filled square) $R e_{x}^{i n i}=10^{8}, \Delta y^{+}=10$; (red solid line, empty triangle) $R e_{x}^{i n i}=10^{6}, \Delta y^{+}=30$. First-order approximations obtained from (17) are plotted with dotted lines aside the corresponding exact solutions.

the limit of infinite Reynolds number $R e_{x}$ however, this ratio asymptotically approaches unity, even though the gap between the reference and the controlled momentum thicknesses, $R e_{\theta}^{r e f}-R e_{\theta}$, grows to infinity. In the light of the analysis conducted in Sec. V, a complementary interpretation for this behavior will be developed.

A decrease of the boundary-layer thickening rate has a detrimental influence on the friction drag: indeed, the thicker the boundary-layer, the weaker the skin-friction. By way of illustration, consider the case of a control with $\Delta y^{+}=10$ and $R e_{x}^{i n i}=10^{6}$. At location $R e_{x}=10^{7}$, where $R e_{\theta}=9.0 \cdot 10^{3}$ and $R e_{\theta}^{r e f}=1.4 \cdot 10^{4}$, the two effects induced by the control are the following:

- a skin-friction reduction with respect to the uncontrolled case at same $R e_{\theta}$. This is the direct effect, addressed in Sec. III. Using Eq. (12) or Fig. 3(b), one gets $D R_{\theta}\left(\operatorname{Re}_{\theta}=9.0 \cdot 10^{3}\right) \approx 45 \%$.

- a decrease of the boundary-layer thickening rate compared to the uncontrolled case. This is the indirect effect. By growing naturally, the uncontrolled boundary-layer friction has felt down to $c_{f}^{r e f}\left(R e_{\theta}^{r e f}=1.4 \cdot 10^{4}\right)=2.37 \cdot 10^{-3}$ while it would have been $9 \%$ higher with the slow boundary-layer growth of the controlled case $c_{f}^{r e f}\left(\operatorname{Re}_{\theta}=9.0 \cdot 10^{3}\right)=2.58 \cdot 10^{-3}$.

When both effects are taken into account, the effective drag benefit is slightly smaller (40\%) than when predicted by taking into account only the first effect.

An additional consequence of the narrowing of the boundary-layer, beneficial for once, has been underlined by Spalart and McLean. ${ }^{18}$ A boundary-layer of reduced displacement thickness will be subject to a smaller viscous pressure drag. Hence, far from fundamental zero-pressure-gradient flat-plate studies, practical applications may take advantage of this indirect effect to reduce their total drag, avoid separation, or even beneficially interact with shock waves.

\section{COMBINED EFFECTS}

It has been shown that the two effects engendered by the control lead to opposite consequences on the drag: the direct effect reduces the skin-friction while the indirect one tends to increase it. One can wonder whether the drag of the controlled boundary-layer at a given location $R e_{x}$ may not finally exceed that of the uncontrolled-but-thicker boundary-layer.

To answer this concern, the drag reduction at fixed $R e_{x}$ must be computed. Using the friction laws (6) and (11) - relating $c_{f}=2 / U_{\infty}^{2}$ and $R e_{\theta}$ - and the boundary-layer growth laws (16) and (17) - relating $R e_{\theta}$ and $R e_{x}$ - the following analytical expressions between $U_{\infty}=\sqrt{2 / c_{f}}$ and $R e_{x}$ can be established:

$$
\operatorname{Re}_{x}^{r e f}\left(U_{\infty}^{+}\right)=\frac{1}{\kappa^{2}}\left(1+\left(\kappa U_{\infty}^{+}-1\right)^{2}\right) e^{\kappa\left(U_{\infty}^{+}-C\right)},
$$




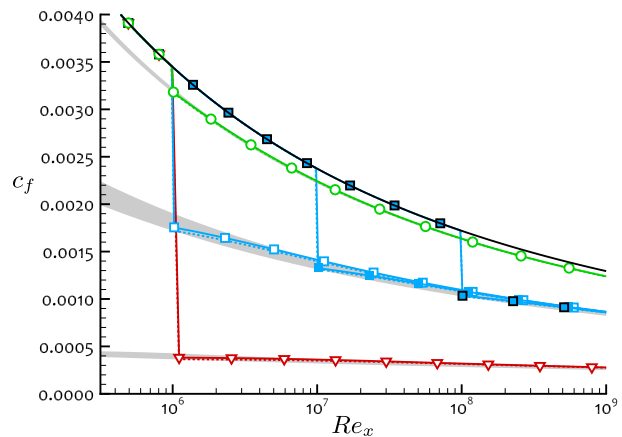

(a)

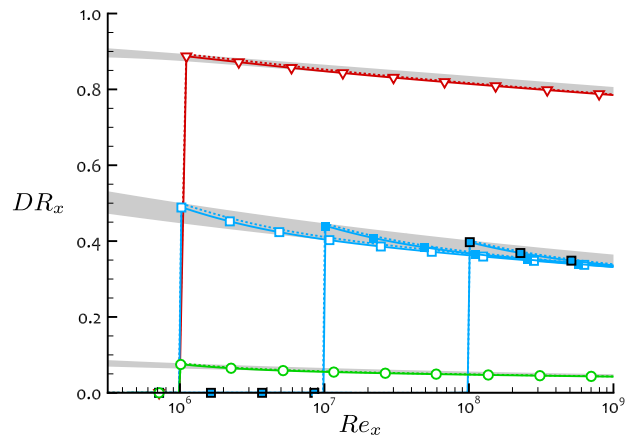

(b)

FIG. 5. Influence of both the damped layer thickness $\Delta y^{+}$and the control starting location $R e_{x}^{i n i}$ on (a) the skin-friction coefficient $c_{f}$ and (b) the drag reduction rate $D R_{x}$ : (black solid line) no control; (green solid line, empty circle) $R e_{x}^{i n i}=10^{6}$, $\Delta y^{+}=1$; (blue solid line, empty square) $R e_{x}^{i n i}=10^{6}, \Delta y^{+}=10$; (blue solid line, filled square) $R e_{x}^{i n i}=10^{7}, \Delta y^{+}=10$; (blue solid line, box filled square) $R e_{x}^{i n i}=10^{8}, \Delta y^{+}=10$; (red solid line, empty triangle) $R e_{x}^{i n i}=10^{6}, \Delta y^{+}=30$. First-order approximations obtained from (17) are plotted with dotted lines aside the corresponding exact solutions. (Shadings) Envelopes obtained from (21) and (22) in which curves for controlled flows must lie whatever $R e_{x}^{i n i}$.

$$
\operatorname{Re}_{x}\left(U_{\infty}^{+}\right) \approx \frac{1}{\kappa^{2}}\left(1+\left(\kappa U_{\infty}^{+}-1\right)^{2}\right) e^{\kappa\left(U_{\infty}^{+}-\Delta y^{+}-C\right)}+\operatorname{Re}_{x}^{o f f s e t}\left(\operatorname{Re}_{\theta}^{i n i}\right),
$$

where $\operatorname{Re}_{x}^{\text {offset }}\left(R e_{\theta}^{i n i}\right)$ has been given in (18). The last formula rests upon the approximations $\Delta y^{+} \ll$ $U_{\infty}^{+2}$ and $\Delta y^{+} \ll \sqrt{R e_{\theta}}$ made to obtain (11) and (17). It is however possible to compute numerically the relation between $R e_{x}$ and $c_{f}$ without approximations using directly (10) and (15). Results are displayed in Fig. 5(a).

Like $D R_{\theta}$, the drag reduction $D R_{x}$ with respect to a given physical abscissa $R e_{x}$ is defined by

$$
D R_{x}\left(R e_{x}\right)=\frac{c_{f}^{r e f}\left(R e_{x}\right)-c_{f}\left(R e_{x}\right)}{c_{f}^{r e f}\left(R e_{x}\right)}=1-\left(\frac{U_{\infty}^{r e f+}\left(R e_{x}\right)}{U_{\infty}^{+}\left(R e_{x}\right)}\right)^{2} .
$$

$D R_{x}$ is represented against $R e_{x}$ in Fig. 5(b) for boundary-layers controlled with different damped layer thicknesses $\Delta y^{+}$and starting locations $R e_{x}^{i n i}$. As expected, the greater $\Delta y^{+}$, the greater the drag reduction. However, counter-intuitively, $c_{f}$ is weaker when the control has been recently applied. Indeed, for a given $\Delta y^{+}$, the latter the control starts - i.e., the higher $\operatorname{Re}_{\theta}^{i n i}$ - the thicker the boundary-layer and thus the weaker the skin-friction $c_{f}$. This reasoning found mathematical justification in (18) and (19b) when considering the location $R e_{x}$ at which a particular level of friction $c_{f}$ will finally be reached.

The two extreme situations are the following:

- the case of a control applied from the very beginning of the boundary-layer: $R e_{\theta}^{i n i} \approx 0$. This case is the one for which the boundary is the thinnest possible, resulting in the highest friction coefficient for a controlled flow, $c_{f, \max }$, and the corresponding lowest inner-scaled free-stream velocity, $U_{\infty, \text { min }}^{+}$. Using (18) and (19b), $U_{\infty, \text { min }}^{+}\left(R e_{x}\right)$ can be implicitly approached by

$$
R e_{x} \approx \frac{1}{\kappa^{2}}\left(\left(\kappa U_{\infty, \min }^{+}-1\right)^{2}+1\right) e^{\kappa\left(U_{\infty, \min }^{+}-\Delta y^{+}-C\right)} .
$$

- the case of a control which has started immediately upstream of the observed location $R e_{x}$. This case is the one for which the boundary is the thickest possible, almost the uncontrolled boundary-layer thickness. Thus, it leads to the lowest skin-friction coefficient for a controlled flow, $c_{f, \min }$, and the corresponding $U_{\infty, \max }^{+}$related to $R e_{x}$ by

$$
R e_{x} \approx \frac{1}{\kappa^{2}}\left(\left(\kappa U_{\infty, \max }^{+}-\kappa \Delta y^{+}-1\right)^{2}+1\right) e^{\kappa\left(U_{\infty, \max }^{+}-\Delta y^{+}-C\right)} .
$$

For each $\Delta y^{+}$, those two limiting cases define an envelope in which the skin-friction of controlled boundary-layers must lie, wherever the control has started. This envelope is represented 
in Fig. 5. It can be observed that, at a given plate location $R e_{x}$, the evolution with $\Delta y^{+}$of the width of that envelope is not monotonic: for instance, at $R e_{x}=10^{6}$, the envelope for $\Delta y^{+}=10$ is wider than for $\Delta y+=1$ and $\Delta y+=30$. The reason for this non-monotonic behavior remains an open question. Even in the less favorable case, that is when $\operatorname{Re} \theta \dot{\theta} \approx 0$, the skin-friction $c_{f, \max }$ of the controlled flow remains below the uncontrolled one $c_{f}^{\text {ref }}$. This answers the previous concern: a controlled boundary-layer, although thinner, always generates less drag at a physical location $x$ than the uncontrolled one. The direct drag-reduction of the control always dominates the detrimental effect of the boundary-layer thickening delay.

To reduce the skin-friction $c_{f}$ at a given location $R e_{x}$, it has been established that the optimal tuning is to make $\Delta y^{+}$as important as possible and to place $R e_{x}^{i n i}$ just upstream the location of interest $R e_{x}$. Are those control parameters also optimal for the reduction of the friction drag accumulated from the beginning of the plate to that location $R e_{x}$ ?

The accumulated skin-friction from the leading edge of a plate is amounted to by the momentum thickness. This is a straightforward result from the integration of (14). Hence, the cumulative effect of drag reduction along the plate is directly visible in Fig. 4. Conclusions stated earlier about the evolution of $R e_{\theta}$ can be brought back with this new perspective: with strong dependence in $\Delta y^{+}$and vanishing influence of $R e_{x}^{i n i}$, the earlier the control is applied, the lower the cumulative drag. When a plate with a given $R e_{x}^{i n i}$ is getting longer, the high skin-friction accumulated before the presence of the control is contributing in a decreasing proportion of the total drag. Having in mind that $\operatorname{Re}_{\theta}$ is proportional to the accumulated drag, this explains both the initial decline of curves $R e_{\theta} / R e_{\theta}^{r e f}$ in Fig. 4(b) and the weakening influence of $R e_{x}^{i n i}$ for further-off station $R e_{x} \gtrsim 10 R e_{x}^{i n i}$. However, the gentle increase of curves in Fig. 4(b) once passed the initial declining transient also has to be interpreted. As mentioned earlier in this section, the drag reduction $D R_{x}$ decreases, although slightly, with the Reynolds number. Hence, at far enough location $R e_{x}$, the friction of the controlled flow is getting only weakly lower than the one of the reference uncontrolled case, meaning that the total drag on the two plates tends to accumulate at almost the same rate from this location. This explains why the ratio between their two accumulated drags approaches asymptotically 1 when $R e_{x} \rightarrow \infty$, even though the gap between them is still slowly increasing and tends towards infinity. Those two seemingly contradictory trends are visually evidenced in Figs. 4(a) and 4(b).

Hence, two somewhat opposite consequences can be underlined: a control applied early $(0 \leftarrow$ $\left.R e_{x}^{i n i}\right)$ minimizes the accumulated drag, while a control applied late $\left(R e_{x}^{i n i} \rightarrow R e_{x}\right)$ minimizes the local drag at downstream locations $R e_{x}$. In both cases, as long as the controlled flow can still be characterized by the composite profile, the bigger $\Delta y^{+}$, the stronger the effect.

Note that in practice, $\Delta y^{+}$cannot be extended infinity. For opposition control, the detection plane must not be located too far from the wall. ${ }^{5}$ The optimal value is found within 10-20 wall units from the wall, where sweeps and ejections occur. With riblets, $\Delta y^{+}$grows linearly with the riblet size until the apparition of Kelvin-Helmholtz-like instabilities. ${ }^{12}$ Those instabilities end up disturbing the flow, such that it cannot be described any more by the composite profile and the drag finally increases. Riblets studied so far hardly outperform $10 \%$ drag-reduction at moderate Reynolds number, $R e_{\theta} \approx O\left(10^{3}\right)$, reflecting an equivalent damped layer thickness around unity.

\section{EFFECT ON THE FIK DECOMPOSITION}

It has been shown that impending turbulence in a tiny near-wall part of the boundary-layer can lead to a considerable drag reduction. In the channel flow case, the drag reduction has been related to a drastic decrease of Reynolds stresses not only in the thin controlled layer but also across the entire boundary-layer extent. ${ }^{11}$

To clarify what link can be made between $c_{f}$ and $R_{i j}$ in the case of a ZPGTBL, the FIK decomposition ${ }^{9}$ is used to split the skin-friction into three terms, respectively, the "laminar," "turbulent," and "spatially heterogeneous" contributions,

$$
c_{f}=c_{f, 1}+c_{f, 2}+c_{f, 3}
$$


with

$$
\begin{aligned}
c_{f, 1} & =\frac{4}{R e_{\delta}} \int_{0}^{\delta} \frac{u}{U_{\infty}} \frac{\mathrm{d} y}{\delta}=\frac{4}{R e_{\delta}}\left(1-\frac{\delta_{1}}{\delta}\right), \\
c_{f, 2} & =4 \int_{0}^{\delta}\left(1-\frac{y}{\delta}\right) \frac{-R_{x y}}{\rho U_{\infty}^{2}} \frac{\mathrm{d} y}{\delta}, \\
c_{f, 3} & =-4 \int_{0}^{\delta} \frac{1}{2}\left(1-\frac{y}{\delta}\right)^{2} \frac{\delta}{\rho U_{\infty}^{2}}\left(I_{x}+\rho \frac{\partial p}{\partial x}\right) \frac{\mathrm{d} y}{\delta}, \\
I_{x} & =\rho u_{i} \frac{\partial u}{\partial x_{i}}-\rho v \frac{\partial^{2} u}{\partial x^{2}}+\frac{\partial R_{x x}}{\partial x} .
\end{aligned}
$$

In a fully developed laminar channel flow, all terms but $c_{f, 1}$ equals zero, hence, the decomposition reduces to its sole "laminar" term. In the case of turbulent channel flow, the "turbulent" term $c_{f, 2}$ also comes into play. Finally, in the present case of spatially developing turbulent boundary-layer, $c_{f, 3}$ accounts for the spatial evolution in the streamwise direction, so none of the three terms vanish.

The scope of this section is to determine to what extent the control influences each of those terms.

\section{A. Laminar contribution $c_{f, 1}$}

The FIK decomposition, and in particular, the "laminar" term $c_{f, 1}$, directly depends on the definition of the boundary-layer thickness. In the case of a channel flow, $\delta$ is unambiguously defined as the channel half-width. For spatially developing boundary-layer, the $99 \%$ free-stream boundary-layer thickness $\delta_{99}$ is customary, but arbitrary, made used of. Nagib et al. ${ }^{16}$ derived its expression by substituting generic outer velocity profile (25) evaluated at $y=\delta_{99}^{\text {ref }}$ into $\delta_{1}$-based Coles-Fernholz relation (26),

$$
\begin{aligned}
u^{r e f+}\left(y^{+}\right) & =\frac{1}{\kappa} \ln \left(y^{+}\right)+B+\frac{\Pi}{\kappa} \mathcal{W}\left(\frac{y^{+}}{\delta^{r e f+}}\right), \\
U_{\infty}^{r e f+} & =\frac{1}{\kappa} \ln \left(\operatorname{Re}_{\delta_{1}}^{r e f}\right)+C_{1},
\end{aligned}
$$

with $B=4.173, \Pi=0.55, \mathcal{W}(0.99) \approx \mathcal{W}(1)=2$, and $C_{1}=3.354$. Using the same methodology, a $100 \%$ free-stream thickness $\delta=\delta_{100}$ can be computed. In order to ease analytical developments in the remaining of this paper, this choice of boundary-layer thickness is used. For the uncontrolled flow, one obtains

$$
\delta^{r e f+}=\operatorname{Re}_{\delta_{1}}^{r e f} / e^{2 \Pi+\kappa\left(B-C_{1}\right)} .
$$

For the composite profile, the boundary-layer thickness satisfies

$$
\begin{aligned}
\delta^{+} & =\delta^{\prime+}+\Delta y^{+} \\
& =\operatorname{Re}_{\delta_{1}^{\prime}}^{\prime} / e^{2 \Pi+\kappa\left(B-C_{1}\right)}+\Delta y^{+} \\
& =\operatorname{Re}_{\delta_{1}} / e^{2 \Pi+\kappa\left(B-C_{1}\right)}+O\left(U_{\infty}^{+} \Delta y^{+}\right),
\end{aligned}
$$

where (2) and (7) has been used.

The laminar contribution $c_{f, 1}$ of a controlled boundary-layer is compared to that of the uncontrolled reference taken at the same $R e_{\delta}$ by

$$
\frac{c_{f, 1}\left(R e_{\delta}\right)}{c_{f, 1}^{r e f}\left(R e_{\delta}\right)}=\frac{R e_{\delta}-R e_{\delta_{1}}\left(R e_{\delta}\right)}{R e_{\delta}-R e_{\delta_{1}}^{r e f}\left(R e_{\delta}\right)},
$$

where $R e_{\delta_{1}}^{r e f}$ and $R e_{\delta_{1}}$ can be expressed as functions of $R e_{\delta}=\delta^{+} U_{\infty}^{+}$from (27) and (28). This yields, after simplifications, 


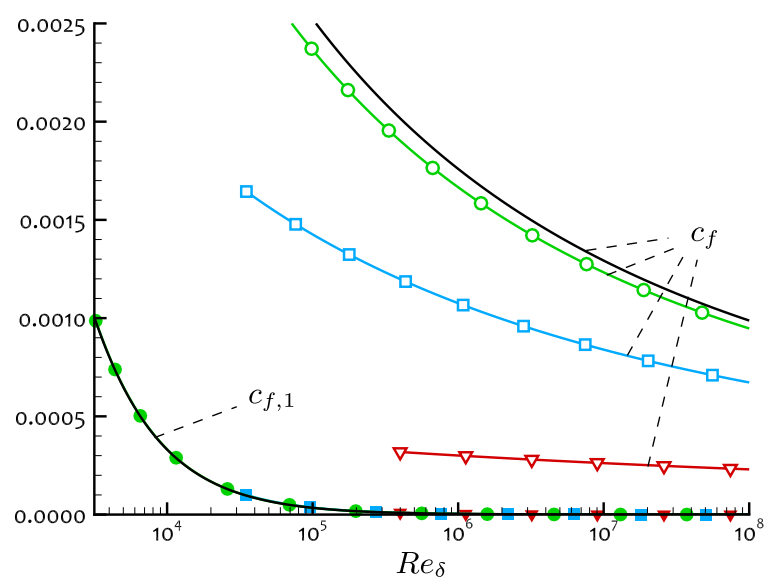

FIG. 6. Influence of the damped layer thickness $\Delta y^{+}$on (empty symbols) the skin-friction coefficient $c_{f}$ and (filled symbols) its laminar contribution $c_{f, 1}$ : (black solid line) no control; (green solid line, circle) $\Delta y^{+}=1$; (blue solid line, square) $\Delta y^{+}=10$; (red solid line, triangle) $\Delta y^{+}=30$. Only data for which $\delta^{+}>100 \Delta y^{+}$are shown.

$$
\begin{aligned}
\frac{c_{f, 1}\left(R e_{\delta}\right)}{c_{f, 1}^{r e f}\left(R e_{\delta}\right)} & =1+\frac{\operatorname{Re}_{\delta_{1}}^{r e f}\left(R e_{\delta}\right)}{\operatorname{Re}_{\delta}-\operatorname{Re}_{\delta_{1}}^{r e f}\left(\operatorname{Re}_{\delta}\right)}\left(1-\frac{U_{\infty}^{r e f+}\left(R e_{\delta}\right)}{U_{\infty}^{+}\left(R e_{\delta}\right)}\right)+O\left(\frac{\Delta y^{+}}{\delta^{+}}\right) \\
& =1+O\left(\frac{\operatorname{Re}_{\delta_{1}}^{r e f}\left(R e_{\delta}\right)}{\operatorname{Re}_{\delta}} D R_{\delta}\left(\operatorname{Re}_{\delta}\right)\right)+O\left(\frac{\Delta y^{+}}{\delta^{+}}\right) .
\end{aligned}
$$

For today's realistic control (for which $D R \lesssim 10 \%$ ) and high enough Reynolds number (such that $\left.R e_{\delta_{1}} \lesssim 0.1 R e_{\delta}\right)$, the laminar skin-friction contribution $c_{f, 1}\left(R e_{\delta}\right)$ can be considered as weakly affected by the control: $c_{f, 1}\left(R e_{\delta}\right) \approx c_{f, 1}^{r e f}\left(R e_{\delta}\right)$. This result is evidenced in Fig. 6, where curves of $c_{f, 1}\left(R e_{\delta}\right)$ for the reference and for all the controlled flows all perfectly overlap.

The "laminar" contribution only depends on the mean velocity profile. Hence, the disturbance of the mean flow induced by the control does not straightforwardly explain the drag reduction, regardless whether controlled and uncontrolled flows are compared at equivalent $R e_{\delta}$ or $R e_{\tau}$ (as evidenced using (27) and (28)).

\section{B. Turbulent contribution $c_{f, 2}$}

The Reynolds stresses of the superimposed turbulent flow, $R_{i j}^{\prime+}\left(y^{\prime+}\right)=R_{i j}^{+}\left(y^{+}>\Delta y^{+}\right)$, have been assumed in Eq. (3b) to follow the profiles $R_{i j}^{r e f+}\left(y^{+}\right)$of the uncontrolled boundary-layer at same Reynolds number $R e_{\tau}$, that is such that: $\delta^{r e f+}=\delta^{\prime+}=\delta^{+}-\Delta y^{+}$. Making explicit the inner scaling, made of $u_{\tau}$ and $l_{\tau}=v / u_{\tau}$ for the controlled flow (respectively, $u_{\tau}^{r e f}$ and $l_{\tau}^{r e f}$ for the reference case), one obtains

$$
\begin{aligned}
& \delta-\Delta y=\left(\frac{l_{\tau}}{l_{\tau}^{r e f}}\right) \delta^{r e f}, \\
& \frac{R_{i j}(y>\Delta y)}{U_{\infty}^{2}}=\left(\frac{U_{\infty}^{r e f+}}{U_{\infty}^{+}}\right)^{2} \frac{R_{i j}^{r e f}\left(\frac{l_{\tau}^{r e f}}{l_{\tau}}(y-\Delta y)\right)}{\left(U_{\infty}^{r e f}\right)^{2}}=\left(\frac{c_{f}}{c_{f}^{r e f}}\right) \frac{R_{i j}^{r e f}\left(\frac{y-\Delta y}{\delta-\Delta y} \delta^{r e f}\right)}{\left(U_{\infty}^{r e f}\right)^{2}} .
\end{aligned}
$$

Substitution into the definition of $c_{f, 2}$ and then change of variable $y \longleftrightarrow \mathrm{y}=\frac{y-\Delta y}{\delta-\Delta y} \delta^{\text {ref }}$ yield

$$
c_{f, 2}=4 \int_{\Delta y}^{\delta}\left(1-\frac{y}{\delta}\right) \frac{c_{f}}{c_{f}^{r e f}} \frac{-R_{x y}^{r e f}\left(\frac{y-\Delta y}{\delta-\Delta y} \delta^{r e f}\right)}{\rho\left(U_{\infty}^{r e f}\right)^{2}} \frac{\mathrm{d} y}{\delta}
$$




$$
\begin{aligned}
& =4\left(1-\frac{\Delta y}{\delta}\right)^{2} \frac{c_{f}}{c_{f}^{r e f}} \int_{0}^{\delta^{r e f}}\left(1-\frac{\mathrm{y}}{\delta^{r e f}}\right) \frac{-R_{x y}^{r e f}(\mathrm{y})}{\rho\left(U_{\infty}^{r e f}\right)^{2}} \frac{\mathrm{dy}}{\delta^{r e f}} \\
& =\left(1-\frac{\Delta y}{\delta}\right)^{2} \frac{c_{f}}{c_{f}^{r e f}} c_{f, 2}^{r e f} .
\end{aligned}
$$

Neglecting $\Delta y^{+} / \delta^{+}$when compared to one, the relative turbulent contribution is conserved,

$$
\frac{c_{f, 2}}{c_{f}}\left(R e_{\tau}\right) \approx \frac{c_{f, 2}^{r e f}}{c_{f}^{r e f}}\left(R e_{\tau}\right) .
$$

Fig. 7 supports this conclusion. The knowledge of $R_{x y}(y)$ and its integration across the boundary-layer were needed to realize this figure. Formulae used so far have been sufficient to approximate global quantities such as $\tau_{w}, \theta$ or $\delta$, but are not to determine the Reynolds shear-stress profile. We decide to overcome this lack using the analytical mean velocity profile $u^{+}\left(y^{+}, U_{\infty}^{+}(x)\right)$ provided by Monkewitz et al. ${ }^{19}$ Along with the following boundary-layer equations, ${ }^{20}$ the mean normal velocity $v(y)$ and then the Reynolds shear-stress $R_{x y}(y)$ can be computed at any location $x$,

$$
\begin{aligned}
\frac{\partial u}{\partial x}+\frac{\partial v}{\partial y} & =0 \\
u \frac{\partial u}{\partial x}+v \frac{\partial u}{\partial y} & =\frac{\partial}{\partial y}\left(v \frac{\partial u}{\partial y}-R_{x y}\right) .
\end{aligned}
$$

The fact that $c_{f, 2} / c_{f}$ is unaltered by the control means that the "turbulent" contribution $c_{f, 2}$ is reduced proportionally to $c_{f}$, that is, greatly reduced. This is surprising at the first sight since $c_{f, 2}$ is defined by an integral of $R_{x y}(y)$, while the turbulent profiles of the controlled flow have been assumed to follow the canonical uncontrolled ones. However, a closer look at Eq. (3b) reveals that controlled and uncontrolled turbulent profiles are equal in wall-unit scaling only. Therefore, the weakening of the turbulent stress intensity is due to a scaling effect caused by the drop of the skin-friction. This explains how, by altering the turbulent fluctuation in a minute near-wall layer, the control spreads its effect across the whole boundary-layer extend.

The same conclusion is reached for channel flows: ${ }^{11}$ among the two non-zero terms $c_{f, 1}$ and $c_{f, 2}$ of the channel flow decomposition, the drag reduction is attributed to a drop of the latter, caused by a weakening of the Reynolds shear-stress.

In actual flows, skin-friction and turbulent stress are intertwined. Does the control first damp turbulence, which alters the momentum transport towards the wall and then reduces the skinfriction? Or, alternatively, does the control first reduce the skin-friction, which diminishes the

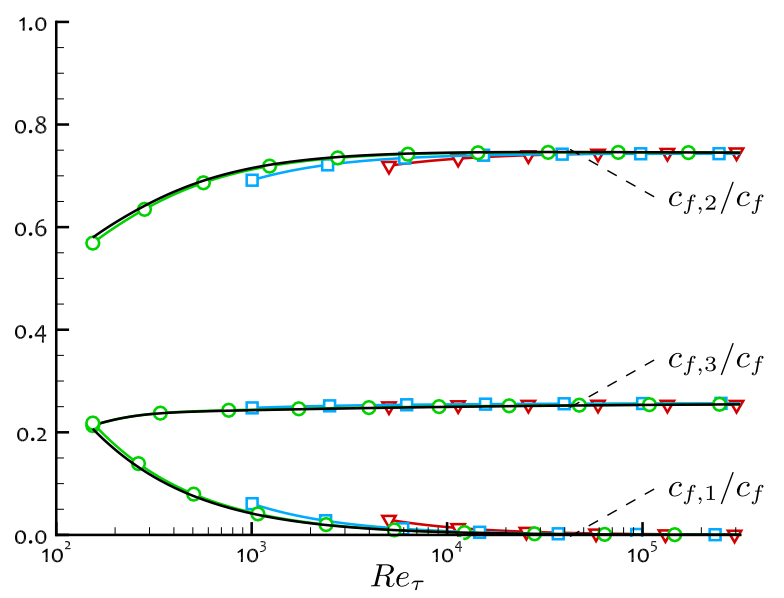

FIG. 7. Influence of the damped layer thickness $\Delta y^{+}$on the relative FIK contribution terms $c_{f, i} / c_{f}$ versus $R e_{\tau}$ : (black solid line) no control; (green solid line, circle) $\Delta y^{+}=1$; (blue solid line, square) $\Delta y^{+}=10$; (red solid line, triangle) $\Delta y^{+}=30$. Only data for which $\delta^{+}>100 \Delta y^{+}$are shown. 
near-wall shear and then weakens the turbulence regeneration process? Depending on the particular drag-reducing device being used (riblets, active feedback control, etc.), physical investigations are necessary to shed light on the specific mechanism in action.

\section{Spatial heterogeneity contribution $c_{f, 3}$}

The last term of the decomposition, $c_{f, 3}$, involves space derivatives in the streamwise direction $x$. Yet, it can be interpreted as the consequence on the skin-friction of the boundary-layer spatial development.

Deck et al. ${ }^{10}$ analyzed the behavior of this term for high-Reynolds-number turbulent boundarylayers and for the Blasius self-similar solution. They noticed that the relative contribution $c_{f, 3} / c_{f}$ remains surprisingly constant and equals approximately $20 \%$ whatever the laminar-versus-turbulent nature of the zero-pressure-gradient flow and its Reynolds number. As evidenced in Fig. 7, the ideal control does not either affect it: $c_{f, 3} / c_{f}$ is found approximately equals to $25 \%$ independently of the presence of the control. This high value is due to the use of the " $100 \%$ free-stream thickness" $\delta_{100}$ defined in (27) instead of the usual $\delta_{99}$. The computation using $\delta=\delta_{99}$ leads to a relative $c_{f, 3}$ contribution of approximately $20.5 \%$, in perfect agreement with the literature.

Even though the boundary-layer development is slowed down by the control, its contribution to the skin-friction still conserves the same weight as for the uncontrolled case. Under the effect of the control, the two major contributions, $c_{f, 2}$ and $c_{f, 3}$, are thus going through a substantial reduction, while the "laminar" contribution $c_{f, 1}$, although lesser at large Reynolds number, is almost unaffected.

\section{COMPARISON WITH REAL DRAG-REDUCING FLOW}

As mentioned in Sec. II, turbulent flows over riblets exhibit velocity and turbulent profiles consistent with those of the composite flow defined here. The riblet design studied by Bannier et al. ${ }^{8}$ leads to a control parameter $\Delta y^{+}$approximately constant on a range of Reynolds numbers extending from $R e_{\theta}=700$ to 1300 and equal to 1.4 . The present analytic study is compared, as a validating test, to the actual turbulent flow developing over the ribbed wall.

The two effects induced by the control-namely, the skin-friction reduction at given $R_{\theta}$ and the decrease of the boundary-layer thickening rate-are represented, respectively, in Figs. 8 and 9. Discrepancies localized at the upstream and downstream ends of the controlled zone $\left(R e_{x}=\right.$ $2.2 \cdot 10^{5}$ and $5.7 \cdot 10^{5}$, corresponding to $R e_{\theta} \approx 640$ and 1300) are due to transitory effects: after the sudden appearance or disappearance of riblets on the wall, the turbulent flow needs a transient

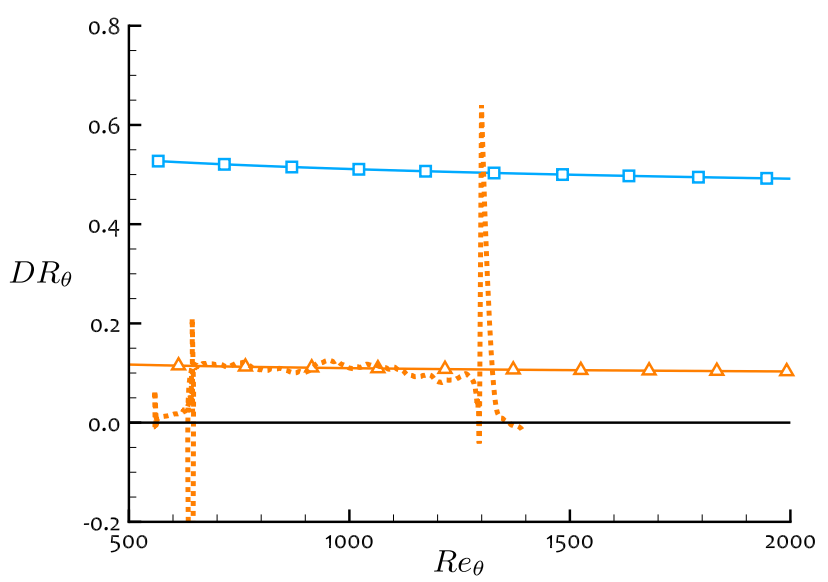

FIG. 8. Drag reduction rate $D R_{\theta}$ versus $R e_{\theta}$. LES riblet-bounded flow (orange dotted line) and analytic composite profile: (orange solid line, triangle) $\Delta y^{+}=1.4$; (blue solid line, square) $\Delta y^{+}=10$. 


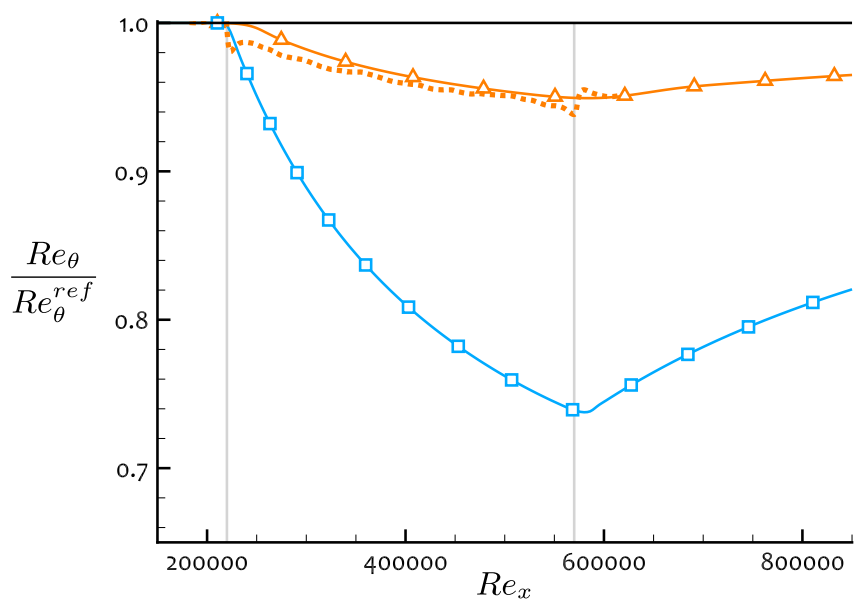

FIG. 9. Momentum thickness Reynolds number ratio $R e_{\theta} / R e_{\theta}^{r e f}$ versus $R e_{x}$. LES riblet-bounded flow (orange dotted line) and analytic composite profile: (orange solid line, triangle) $\Delta y^{+}=1.4$; (blue solid line, square) $\Delta y^{+}=10$. Vertical lines indicate the upstream $\left(R e_{x}^{i n i}=2.2 \cdot 10^{5}\right)$ and downstream $\left(R e_{x}^{\text {end }}=5.7 \cdot 10^{5}\right)$ ends of the controlled zone.

to re-adapt and reach a new equilibrium. Not before such equilibrium is reached, the flow is well portrayed by the composite profile (see Fig. 2). Both the drag reduction and the boundary-layer development are in that case well predicted by the present analytical model. The case $\Delta y^{+}=10$ is represented so as to put into perspective the saving that could potentially be obtained, on this Reynolds number range, with a hypothetical control device that would damp fluctuations within the ten first wall-units from the wall.

Considering simultaneously the two previous effects, the evolution of the skin-friction along the streamwise direction $R e_{x}$ is shown in Fig. 10, and the relative contributions of the FIK terms in Fig. 11. Those results complete the validation of the analytical model, outside of the transient areas localized at both ends of the controlled zone.

Coles-Fernholz correlation (6), on which is based this analytical development, and Monkewitz et al.'s asymptotic velocity profile, ${ }^{19}$ used to compute FIK terms, successfully describe the present flows, even though the considered Reynolds numbers are below their recommended range of application $\left(\operatorname{Re}_{\theta}=O\left(10^{3}\right)\right)$.

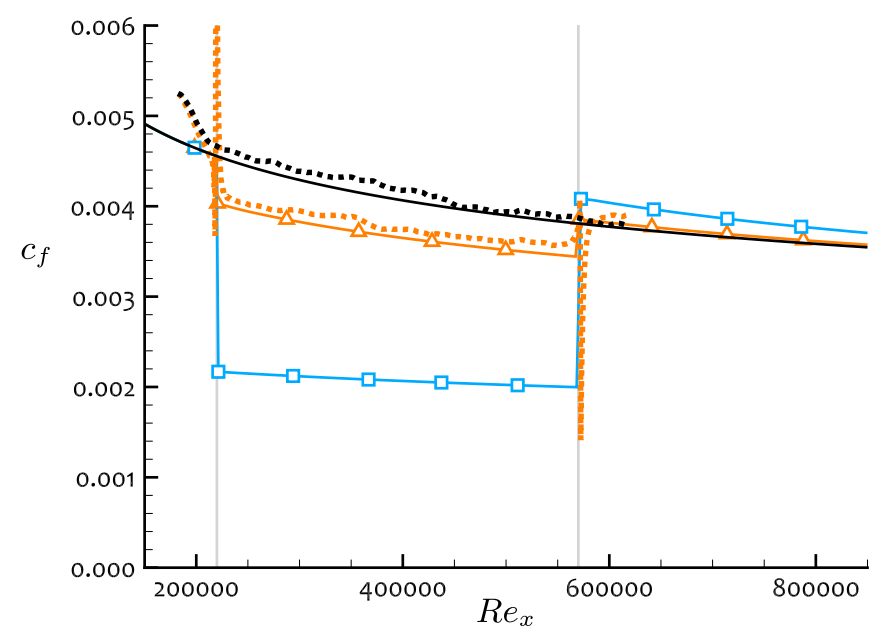

FIG. 10. Skin friction coefficient $c_{f}$ versus $R e_{x}$. LES simulations: (black dotted line) reference case; (orange dotted line) riblet case. Analytic composite profile: (black solid line) no control; (orange solid line, triangle) $\Delta y^{+}=1.4$; (blue solid line, square) $\Delta y^{+}=10$. Vertical lines indicate the upstream $\left(R e_{x}^{i n i}=2.2 \cdot 10^{5}\right)$ and downstream $\left(R e_{x}^{\text {end }}=5.7 \cdot 10^{5}\right)$ ends of the controlled zone. 


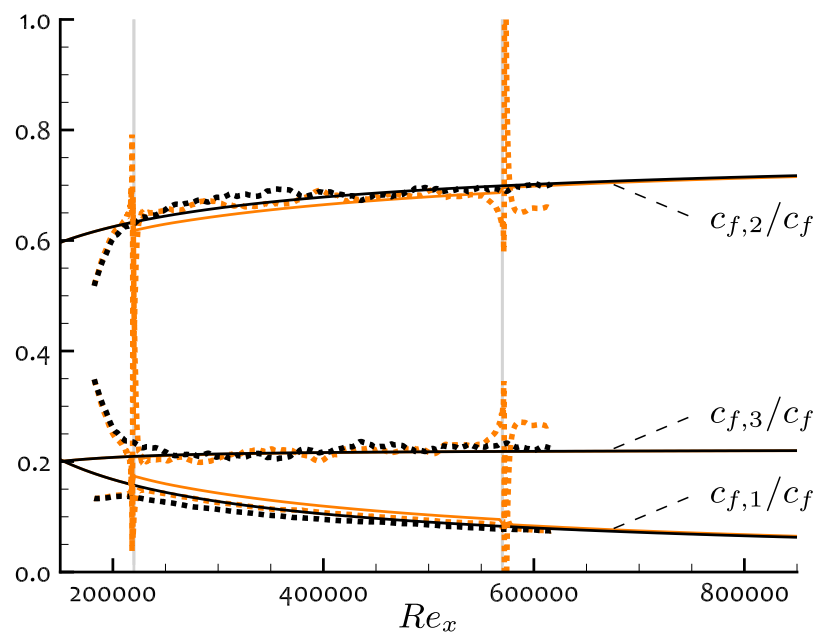

FIG. 11. Relative contribution $c_{f, i} / c_{f}$ of the FIK decomposition terms versus $R e_{x}$. LES simulations: (black dotted line) reference case; (orange dotted line) riblet case. Analytic composite profile: (black solid line) no control; (orange solid line) $\Delta y^{+}=1.4$. Vertical lines indicate the upstream $\left(\operatorname{Re} e_{x}^{i n i}=2.2 \cdot 10^{5}\right)$ and downstream $\left(\operatorname{Re} e_{x}^{\text {end }}=5.7 \cdot 10^{5}\right)$ ends of the controlled zone.

\section{CONCLUSIONS}

Analytical formulae have been derived to describe the friction and the spatial development of zero-pressure-gradient turbulent boundary-layers for which near-wall turbulent fluctuations are supposed to be ideally damped. Under such control, the assumed velocity profiles are in good agreement with the ones actually observed for riblet-controlled flows, making riblets a practical and realistic benchmark to compare our analytical results.

Two main effects have been isolated to characterize the action of the control. First, the skinfriction is substantially reduced when compared to an uncontrolled flow at similar Reynolds number $R_{\theta}$. A similar effect is observed in fully developed turbulent channel flows under analogous control. The second effect, which is a direct consequence of the skin-friction drop, is a decrease of the boundary-layer spatial development rate. By growing more slowly, the boundary-layer thickness is thinner than when uncontrolled, which has detrimental effect on its drag. Despite that penalty, controlled flows at any given streamwise location $x$ have been shown to always generate less drag than their uncontrolled counterpart.

The FIK decomposition splits the skin-friction into contributions to provide physical insight on its origin. While the laminar term is evidenced to be unaffected by the control, the turbulent contribution is as much reduced as the skin-friction itself. A wall-unit scaling effect is responsible for the weakening of turbulent intensities, resulting in the drop of the turbulent contribution. The same conclusion has already been drawn for channel flows subject to similar control. However, in the present case of spatially developing boundary-layer, the FIK contribution resulting from the spatial heterogeneity also comes into play. Its relative contribution to the skin-friction, known to be independent from the state of the flow and from the Reynolds number, is also found unaltered by the presence of the control.

Thanks to the influence of the control on those two major drag contributors, significant drag reduction is still achievable for high Reynolds number flows, even though the manipulated near-wall layer tends to represent an infinitesimal part of the boundary-layer. Because the control influences the boundary-layer growth, the local drag-reduction is maximized when the actuation starts just upstream. Conversely, a control applied early minimizes the global cumulative drag.

\section{ACKNOWLEDGMENTS}

This work was granted access to the HPC resources from IDRIS under the Allocation No. 2015-i20152a7192 made by the GENCI. The financial support from Ecole Polytechnique to the thesis of Amaury Bannier is gratefully acknowledged. 
${ }^{1}$ M. J. Walsh and L. M. Weinstein, "Drag and heat transfer on surfaces with small longitudinal fins,” AIAA Paper No. 78-1161, 1978.

${ }^{2}$ R. García-Mayoral and J. Jiménez, "Drag reduction by riblets," Philos. Trans. R. Soc., A 369, 1412-1427 (2011).

${ }^{3}$ T. Min and J. Kim, "Effects of hydrophobic surface on skin-friction drag," Phys. Fluids 16, L55 (2004).

${ }^{4}$ J. P. Rothstein, "Slip on superhydrophobic surfaces," Annu. Rev. Fluid Mech. 42, 89-109 (2010).

${ }^{5}$ H. Choi, P. Moin, and J. Kim, “Active turbulence control for drag-reduction in wall-bounded flows," J. Fluid Mech. 262, 75-110 (1994).

${ }^{6}$ N. Kasagi, Y. Suzuki, and K. Fukagata, "Microelectromechanical systems-based feedback control of turbulence for skin friction reduction," Annu. Rev. Fluid Mech. 41, 231-251 (2009).

${ }^{7}$ Q.-J. Xia, W.-X. Huang, C.-X. Xu, and G.-X. Cui, "Direct numerical simulation of spatially developing turbulent boundary layers with opposition control," Fluid Dyn. Res. 47, 025503 (2015).

${ }^{8}$ A. Bannier, E. Garnier, and P. Sagaut, "Riblet flow model based on an extended FIK identity," Flow, Turbul. Combust. 95, 351-376 (2015).

${ }^{9}$ K. Fukagata, K. Iwamoto, and N. Kasagi, "Contribution of Reynolds stress distribution to the skin friction in wall-bounded flows," Phys. Fluids 14, L73 (2002).

${ }^{10}$ S. Deck, N. Renard, R. Laraufie, and P.-E. Weiss, "Large-scale contribution to mean wall shear stress in high-Reynoldsnumber flat-plate boundary layers up to $\mathrm{Re}_{\delta}=13650$," J. Fluid Mech. 743, 202-248 (2014).

${ }^{11}$ K. Iwamoto, K. Fukagata, N. Kasagi, and Y. Suzuki, "Friction drag reduction achievable by near-wall turbulence manipulation at high Reynolds numbers," Phys. Fluids 17, 011702 (2005).

${ }^{12}$ R. García-Mayoral and J. Jiménez, "Hydrodynamic stability and breakdown of the viscous regime over riblets," J. Fluid Mech. 678, 317-347 (2011).

${ }^{13}$ C. Lee, J. Kim, and H. Choi, “Suboptimal control of turbulent channel flow for drag reduction,” J. Fluid Mech. 358, 245-258 (1998).

${ }^{14}$ T. R. Bewley, P. Moin, and R. Temam, "DNS-based predictive control of turbulence: An optimal benchmark for feedback algorithms," J. Fluid Mech. 447, 179-225 (2001).

${ }^{15}$ H.-H. Fernholz and P. J. Finley, "The incompressible zero-pressure-gradient turbulent boundary layer: An assessment of the data," Prog. Aeronaut. Sci. 32, 245-311 (1996).

${ }^{16}$ H. M. Nagib, K. A. Chauhan, and P. A. Monkewitz, "Approach to an asymptotic state for zero pressure gradient turbulent boundary layer," Philos. Trans. R. Soc., A 365, 755-770 (2007).

${ }^{17}$ H. Schlichting, Boundary-Layer Theory, McGraw-Hill Series in Mechanical Engineering (McGraw-Hill, New York, 1979).

${ }^{18}$ P. R. Spalart and J. D. McLean, "Drag reduction: Enticing turbulence, and then an industry," Philos. Trans. R. Soc., A 369, 1556-1569 (2011).

${ }^{19}$ P. A. Monkewitz, K. A. Chauhan, and H. M. Nagib, "Self-consistent high-Reynolds-number asymptotics for zero-pressuregradient turbulent boundary layers," Phys. Fluids 19, 115101 (2007).

20 J. Cousteix, Turbulence et Couche Limite (Cépadués, 1989). 